\title{
Triple immunofluorescence labeling of atherosclerotic plaque components in apoE/LDLR ${ }^{-/-}$mice
}

\author{
Mariusz Gajda $^{1}$, Jacek Jawień ${ }^{2}$, Lukasz Mateuszuk ${ }^{2}$, Grzegorz J. Lis ${ }^{1}$, \\ Andrzej Radziszewski ${ }^{3}$, Stefan Chlopicki ${ }^{2}$, Jan A. Litwin ${ }^{1}$
}

Departments of ${ }^{1}$ Histology, ${ }^{2}$ Experimental Pharmacology, Chair of Pharmacology and ${ }^{3}$ Nephrology, Jagiellonian University Medical College, Kraków, Poland

\begin{abstract}
This paper presents a simple and reliable method of triple immunofluorescence staining that allows simultaneous detection of various cell types present in atherosclerotic plaque of apolipoprotein E and LDL receptor-double knockout (apoE/LDLR ${ }^{-/}$) mice. We used combined direct and indirect procedures applying commercially available primary antibodies raised in different species to detect smooth muscle cells (Cy3-conjugated mouse anti-smooth muscle actin, SMA), macrophages (rat anti-CD68) and T lymphocytes (rabbit anti-CD3). Fixation of the material in acetone and modified incubation protocol employing nonfat dry milk in preincubation and incubation media significantly increased the intensity of labeling and effectively quenched the background. Our method offers an efficient way to detect qualitative as well as quantitative changes of macrophages, T lymphocytes and smooth muscle cells in atherosclerotic plaque of apoE/LDLR ${ }^{-/-} \mathrm{mice}^{-}$ during atherosclerosis development or in response to pharmacological treatment.
\end{abstract}

Key words: Immunohistochemistry - Smooth muscle actin - CD3 - CD68 - apoE/LDLR-double knockout mice Atherosclerosis

\section{Introduction}

Gene-targeting in mice has become a perfect tool to experimentally reproduce human diseases. In particular, apolipoprotein E (apoE) and LDL receptor-double knockout (apoE/LDLR ${ }^{-/}$) mice spontaneously develop severe hypercholesterolemia and atherosclerotic plaques [1]. In the light of inflammatory theory of atherosclerosis, immune and inflammatory cells including macrophages and lymphocytes are involved in plaque formation [2]. Another component of the plaque, smooth muscle cells from the vascular media, proliferate and migrate into the lesion area [3]. It is now clear that activation of macrophages, $\mathrm{T}$ lymphocytes as well as smooth muscle cells determines the outcome of the atherosclerosis [4]. Hence, good methods of assessment of the distribution and abundance of these three cell types in the plaque are mandatory for better understanding of multicellular mechanisms involved in the

Correspondence: M. Gajda, Dept. Histology, Jagiellonian University Medical College, Kopernika 7, 31-034 Kraków, Poland; tel.: (+4812) 4227027, fax.: (+4812) 4227027,

e-mail:mmgajda@cyf-kr.edu.pl plaque formation and for estimation of the anti-atherosclerotic treatment effects. Still, in the vast majority of studies on experimental atherosclerosis in gene-targeted animals, single immunostaining for CD68, SMA or for other antigens has been used [5-7], since simultaneous detection of multiple cell-bound antigens frequently represents a technical challenge. Different affinity of the used antisera to their target antigens and to other tissue components to which they can bind nonspecifically, as well as possible interactions between antisera present in the same incubation medium often results in such unwanted effects as low intensity of staining and/or high background. In the present study we have attempted to overcome these limitations by creating a simple and reliable method of triple immunostaining of smooth muscle cells, macrophages and $\mathrm{T}$ lymphocytes designed for routine qualitative and quantitative assessment of the content of atherosclerotic plaques in apoE/LDLR ${ }^{-/}$mice.

\section{Materials and methods}

Animals. Ten female apoE $\mathrm{E}^{-/ /} \mathrm{LDLR}^{-/}$mice on the mixed C57BL/6J $\times 129 / \mathrm{SvJ}$ background were obtained from Taconic (Ejby, Denmark). Mice were housed at $12 \mathrm{~h}$ light/dark cycle in air- 

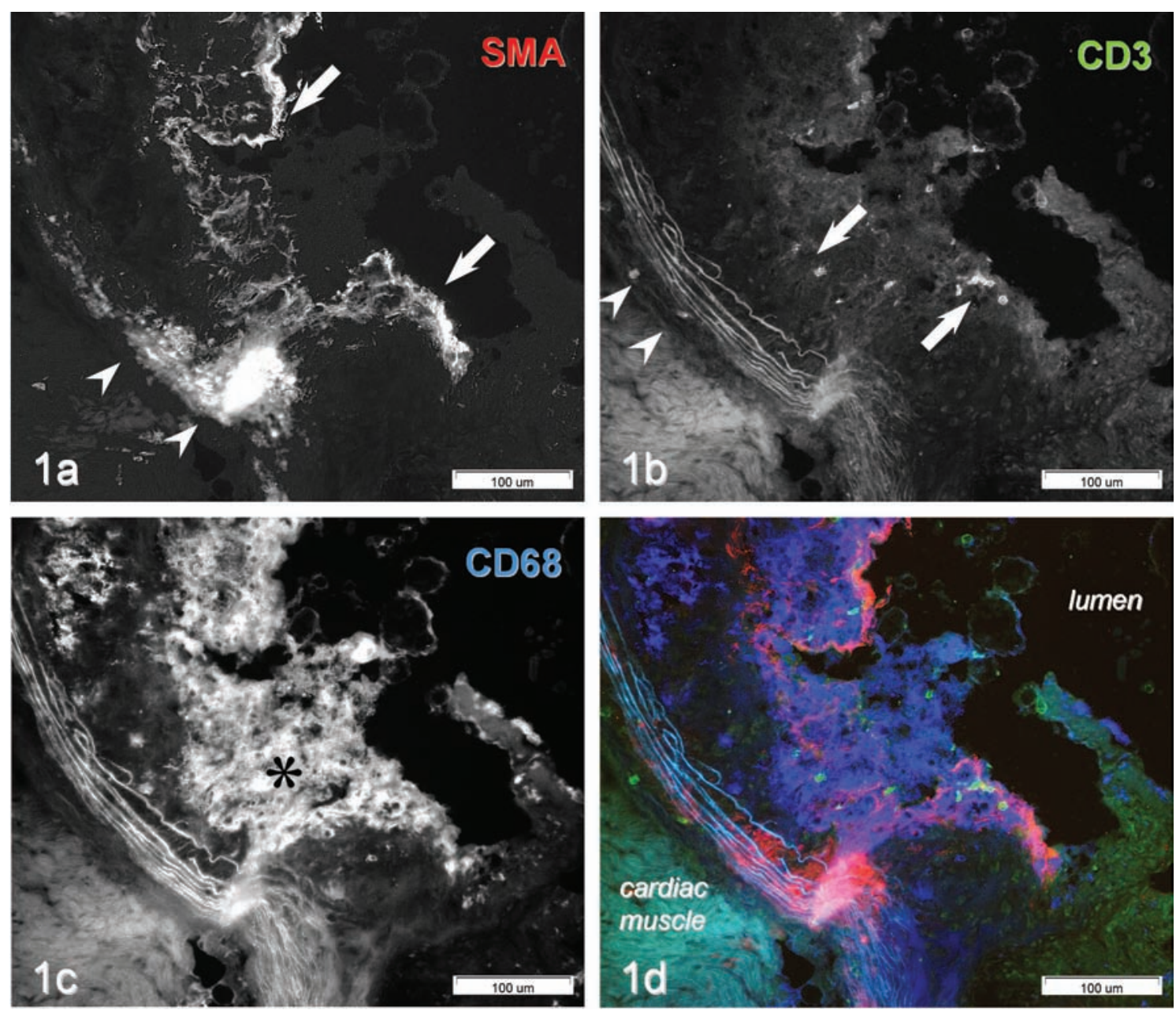

Fig. 1. Triple immunofluorescence of apoE/LDLR ${ }^{-/}$mouse aortic root. The figure presents immunoreactivities for SMA (a), CD3 (b) and CD68 (c), as well as combined image showing in color all three recorded channels (d). Abundant smooth muscle cells are located in the atherosclerotic plaque (a, arrows) and in normal smooth musculature of the vascular media (a, arrowheads). T lymphocytes are present in the plaque (b, arrows) and in the adventitia (b, arrowheads). A massive infiltration of macrophages (c, asterisk) can be observed in the plaque.

conditioned rooms $\left(22.5 \pm 0.5^{\circ} \mathrm{C}, 50 \pm 5 \%\right.$ humidity $)$ and had an unlimited access to water and standard chow diet. Six months-old mice were sacrificed by intraperitoneal administration of sodium thiopental (Vetbutal, Biowet, Puławy, Poland; $100 \mathrm{mg} / \mathrm{kg}$ b.w.).

Specimen preparation. In anesthetized mice, thorax was longitudinally opened, right atrium was incised and the heart was perfused with phosphate buffered saline (PBS, $\mathrm{pH}=7.4$ ) through the apex of left ventricle. Heart was dissected out, rinsed with PBS, embedded in OCT compound (Cell Path, Oxford, UK) and snap-frozen. Ten $\mu \mathrm{m}$-thick frozen sections were cut according to a standardized protocol (for details see [8]). Briefly, serial sections from the proximal $1 \mathrm{~mm}$ of the aortic root were collected on separate slides, air dried and fixed for $10 \mathrm{~min}$ either in acetone or in $4 \%$ phosphate-buffered paraformaldehyde $(\mathrm{pH}=7.4)$ at room temperature.

Immunohistochemistry. A preincubation step with 5\% normal goat serum (Vector, Burlingame, CA; \# S-1000) in PBS containing $0.01 \%$ sodium azide, $0.05 \%$ thimerosal, $0.1 \%$ bovine serum albumin, $0.5 \%$ Triton X-100 with or without $2 \%$ nonfat dry milk powder (SM Gostyń, Poland) was applied for 40 min to reduce nonspecific binding and to increase penetration of the antibodies. For simultaneous demonstration of three antigens, combined direct and indirect triple-staining immunofluorescence procedure using pri- mary antibodies raised in different species was applied. The sections were incubated overnight in humid chambers at room temperature with mixture of three primary antibodies: (1) Cy3-conjugated mouse anti- $\alpha$-smooth muscle actin (SMA; Sigma-Aldrich, St. Louis, MO; \# C6198; diluted 1:800), (2) rat anti-mouse CD68 (Serotec, Oxford, UK; \# MCA1957XZ; diluted 1:800) and (3) rabbit anti-CD3 (Calbiochem, Darmstadt, Germany; \# PC630; diluted 1:600). After rinsing in PBS, sections were incubated for $30 \mathrm{~min}$ at room temperature with goat anti-rat IgG biotinylated antiserum (Jackson IR, West Grove, PA; \# 112-065-143; diluted 1:800) and Cy2-conjugated goat anti-rabbit antiserum (Jackson IR; \# 111-225144; diluted 1:500). Following another rinse in PBS, AMCA-conjugated streptavidin (Vector; \# SA-5008; diluted 1:200) was applied for $1 \mathrm{~h}$ at room temperature. After final rinse in PBS, the sections were mounted in Vectashield medium (Vector; \# H-1000) to minimize photobleaching of fluorochromes.

Primary and secondary antisera were diluted in the solution used for preincubation, while streptavidin conjugate was dissolved in PBS. In the controls, the primary or secondary antibodies were omitted and replaced by nonimmune serum. All chemical reagents were obtained from Sigma-Aldrich unless specified otherwise in the text.

Microscopic examination. Olympus BX50 epifluorescence microscope (Olympus, Tokyo, Japan) equipped with mercuric 


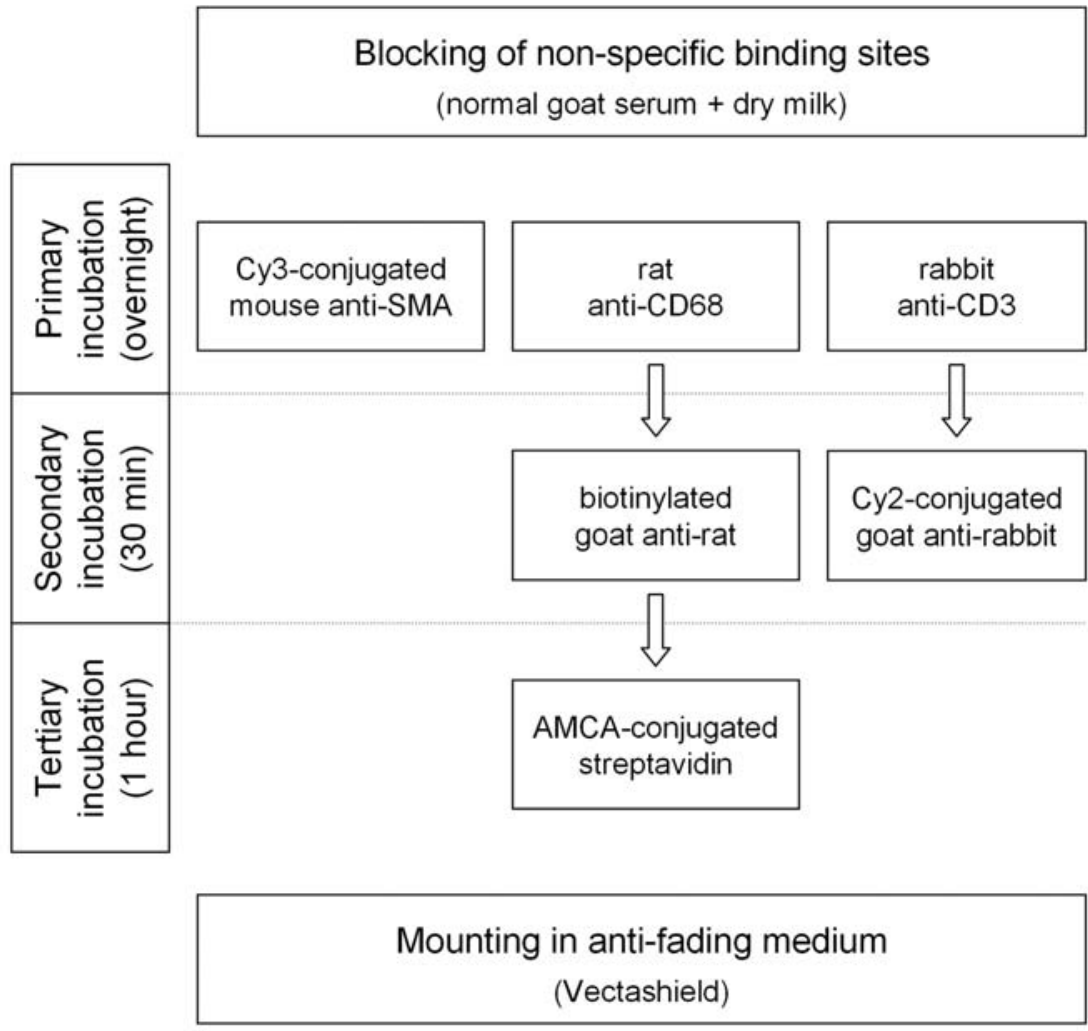

Fig. 2. Block diagram showing the stages of triple immunostaining protocol used in the study. burner and filter sets: U-MNG, U-MNIBA and U-MWA for detection of $\mathrm{Cy} 3$ (red), Cy2 (green) and AMCA (blue) fluorescence, respectively, was used to examine the immunostaining. Images of the aortic roots were recorded using Olympus DP71 digital CCD camera, stored as TIFF files and processed using Olympus AnalySIS FIVE software.

Ethical issues. National and international principles of laboratory animal care were followed and all procedures were approved by and conformed to guidelines of the Jagiellonian University Animal Experimentation Ethics Committee.

\section{Results}

\section{Technical issues}

The intensity of immunofluorescence for all antigens employed was considerably higher in sections fixed in acetone as compared with those fixed in paraformaldehyde. While using standard antibody dilutor without dry milk, we observed very high background for CD68 immunostaining (green channel). After addition of 2\% nonfat dry milk to the preincubation and incubation media, the intensity of nonspecific background staining for CD68 was significantly lower in the aortic root and atherosclerotic plaque, however, the adjacent cardiac muscle still displayed a relatively high background (Fig. 1b-d). Moreover, intense autofluorescence of elastic fibers present in aortic media was seen in green and blue channels (Fig. 1b-d). The controls showed no immunostaining. A simple diagram illustrating the applied protocol is depicted in Figure 2.

\section{Immunolabeling of atherosclerotic plaque cells}

In aortic roots of 6 months-old apoE/LDLR ${ }^{-/-}$mice fed a standard diet, advanced atherosclerotic lesions were observed. CD68 immunostaining showed massive accumulation of macrophages (Fig. 1a,d). They were located not only in the shoulders of atherosclerotic lesions but were also abundant in the central (major) region of the plaque, known to contain rich lipid deposits. Smooth muscle actin (SMA) immunoreactivity was seen in normal smooth muscle cells present in the media of the aortic root as well as in smooth muscle cells near the surface of the atherosclerotic lesion (Fig. 1c,d). The autofluorescence of elastic fibers present in media was seen in green and blue channels (Fig. 1b-d).

In the plaques, CD3-immunopositive $\mathrm{T}$ lymphocytes were occasionally seen in regions located close to the vessel lumen (Fig. 1b,d). Numerous T cells were also present in the adventitia.

\section{Discussion}

The protocol designed for routine identification of three cell populations in the atherosclerotic plaque should be relatively simple and reliable. We chose fixation in acetone which is generally regarded as the fixative of choice for immunostaining of frozen sections [9], although some investigators reported better results with paraformaldehyde [10]. In case of atheroma 
material from apoE/LDLR ${ }^{-/}$mice, acetone fixation was clearly superior to formaldehyde in terms of antigen preservation, yielding higher intensity of immunofluorescence. Cross-linking fixatives were shown to better preserve tissue structure but covalent modifications of antigen determinants may abolish antibody binding [10]. However, adjacent sections collected on separate slides can be fixed in paraformaldehyde and used for other stainings [6]. Another advantage of paraformaldehyde fixation is that sections can be stored for longer time than those fixed in acetone.

Our protocol employs commercially available antibodies and fluorescent labels which make possible examination of the specimen in both, conventional and confocal microscopes.

We used primary antibodies raised in different species that allow to adapt a simple immunohistochemical protocol. Alternatively, antibodies from the same species can be used but the staining procedure requires involvement of more complicated techniques such as tyramide signal amplification and monovalent Fab antibodies [11]. Apart from increased probability of technical failure, such method seems to be less useful for routine evaluation. On the other hand, laboratories which have successful experience with a set of antisera from the same species, will find such protocol as their protocol of choice.

Application of the direct immunohistochemical technique eliminates problems with interactions of the secondary antibodies, especially when the primary antisera originate from the same species as the host material (what is the case in the present study). However, satisfactory immunostaining with directly labeled antibodies with no need for signal amplification can be obtained only when the concentration of the target antigen is sufficiently high. That is why smooth muscle actin can easily be detected by direct immunofluorescence. For the remaining two antigens demonstrated by indirect technique, in order to minimize possible interactions, we choose two different mechanisms of secondary antibody detection: Cy2conjugated secondary antibody for CD3 and biotinylated antiserum plus AMCA-labeled streptavidin for CD68. This selection of immunohistochemical techniques allowed a clear discrimination of the studied cell populations, although other detection systems may also be applied.

Nonfat dry milk is a well known, cheap and commonly available blocking reagent used to prevent nonspecific background staining [12]. In our hands it has also proven effective, quenching the background in the vascular wall and in atheromas.

A strong autofluorescence of elastic fibers is a well known phenomenon which was also present in our material. To avoid this disadvantage, immuonoenzy- matic reaction instead of immunofluorescence might be applied. However, immunoenzymatic techniques give much worse results in multiple labeling than immunofluorescence.

In summary, the protocol described in this paper can be recommended as a routine identification method for principal cell populations in the atherosclerotic plaque. The presented procedure can be used in studies evaluating effects of pharmacological compounds and diets on the development and composition of atherosclerotic lesions in gene-targeted mouse models.

Acknowledgements: Financial support was provided by a statutory grant K/ZDS/000685 from the Jagiellonian University Medical College to M.G.

\section{References}

[ 1] Ishibashi S, Herz J, Maeda N, Goldstein JL, Brown MS. The two-receptor model of lipoprotein clearance: tests of the hypothesis in "knockout" mice lacking the low density lipoprotein receptor, apolipoprotein E, or both proteins. Proc Natl Acad Sci U S A. 1994;91:4431-4435.

[2] Hansson GK. Inflammation, atherosclerosis, and coronary artery disease. $N$ Engl J Med. 2005;352:1685-1695.

[3] Willis AI, Pierre-Paul D, Sumpio BE, Gahtan V. Vascular smooth muscle cell migration: current research and clinical implications. Vasc Endovascular Surg. 2004;38:11-23.

[4] Kaperonis EA, Liapis CD, Kakisis JD, Dimitroulis D, Papavassiliou VG. Inflammation and atherosclerosis. Eur $J$ Vasc Endovasc Surg. 2006;31:386-393.

[ 5] Elhage R, Jawien J, Rudling M, et al. Reduced atherosclerosis in interleukin-18 deficient apolipoprotein E-knockout mice. Cardiovasc Res. 2003;59:234-240.

[ 6] Jawien J, Gajda M, Rudling M, et al. Inhibition of five lipoxygenase activating protein (FLAP) by MK-886 decreases atherosclerosis in apoE/LDLR-double knockout mice. Eur J Clin Invest. 2006;36:141-146.

[7] Robertson AK, Rudling M, Zhou X, Gorelik L, Flavell RA, Hansson GK. Disruption of TGF-beta signaling in T cells accelerates atherosclerosis. J Clin Invest. 2003;112:13421350 .

[ 8] Nicoletti A, Kaveri S, Caligiuri G, Bariety J, Hansson GK. Immunoglobulin treatment reduces atherosclerosis in apo $\mathrm{E}$ knockout mice. J Clin Invest. 1998;102:910-918.

[9] Richter T, Nahrig J, Komminoth P, Kowolik J, Werner M. Protocol for ultrarapid immunostaining of frozen sections. $J$ Clin Pathol. 1999;52:461-463.

[10] Cinar O, Semiz O, Can A. A microscopic survey on the efficiency of well-known routine chemical fixatives on cryosections. Acta Histochem. 2006;108:487-496.

[11] Brouns I, Van Nassauw L, Van Genechten J, et al. Triple immunofluorescence staining with antibodies raised in the same species to study the complex innervation pattern of intrapulmonary chemoreceptors. $J$ Histochem Cytochem. 2002;50:575-582.

[12] Duhamel RC, Johnson DA. Use of nonfat dry milk to block nonspecific nuclear and membrane staining by avidin conjugates. J Histochem Cytochem. 1985;33:711-714.

Submitted: 30 October, 2007 Accepted after reviews:28 January, 2008 